\title{
Predicting poor short- and medium-term survival after transcatheter aortic valve implantation: a single UK centre experience
}

\author{
Authors: Zong Xuan Lee, ${ }^{A}$ Senthil Elangovan, ${ }^{B}$ Richard Anderson ${ }^{B}$ and Peter Groves ${ }^{B}$
}

\section{Introduction}

Transcatheter aortic valve implantation (TAVI) for severe, symptomatic aortic stenosis improves quality of life and survival in most patients. It is, however, important to identify patients who are unlikely to get these benefits from TAVI so that futile treatment can be avoided. Futility in this context can be regarded as lack of functional improvement or death within the first 2 years after the procedure. ${ }^{1}$ The FRANCE-2 multiparametric risk score was previously developed to predict mortality after TAVI and comprises nine pre-procedural factors integrated into a 21-point scoring system. ${ }^{2}$ The FRANCE-2 score was originally validated against early (up to 30 days) mortality after TAVI but its value in anticipating longer term outcomes is uncertain.

\section{Aims}

The aims of this study were to determine whether the FRANCE-2 scoring system is of value in determining medium- as well as shortterm survival in patients undergoing TAVI in a single UK centre, and to compare its relative merits in this regard with the logistic EuroSCORE.

\section{Methods}

A cohort of 187 consecutive patients undergoing TAVI in a single UK centre were studied. Baseline clinical data were collected from the UK Central Cardiac Audit Database (CCAD) and patient records. Mortality tracking was achieved in $100 \%$ of patients. FRANCE-2 risk scores were calculated retrospectively and c-statistics were applied to determine the discriminative power of the FRANCE-2 score and the logistic EuroSCORE in associating with mortality. Using the FRANCE-2 scores, the patients were divided into low-risk (score 0), moderate-risk (score 1-5) and high-risk (score >5) groups and the survival outcomes were compared.

\section{Results}

Of the 187 patients, $57.2 \%$ were male and the mean age was $80.9 \pm 6.9$ years. Survival rates after TAVI at 30 days, 1 - and

Authors: ${ }^{\text {A } C a r d i f f ~ U n i v e r s i t y, ~ C a r d i f f, ~ W a l e s, ~ U K ; ~}{ }^{B}$ University Hospital of Wales, Cardiff, Wales, UK

\begin{tabular}{|c|c|}
\hline Patient parameters & Values \\
\hline Age $\geq 90$ years & $7.0 \%(n=13)$ \\
\hline BMI $<18.5$ & $1.6 \%(n=3)$ \\
\hline New York Heart Association Class IV & $7.5 \%(n=14)$ \\
\hline Acute pulmonary oedema $\geq 2$ in past year & $6.4 \%(n=12)$ \\
\hline Systolic pulmonary artery pressure $\geq 60 \mathrm{mmHg}$ & $5.9 \%(n=11)$ \\
\hline Critical preoperative state & $4.3 \%(n=8)$ \\
\hline Respiratory insufficiency & $43.9 \%(n=82)$ \\
\hline Dialysis & $1.1 \%(n=2)$ \\
\hline $\begin{array}{l}\text { Delivery approach other than transfemoral or } \\
\text { subclavian route }\end{array}$ & $0 \%(n=0)$ \\
\hline
\end{tabular}

2-years were 95.7\% ( $n=179), 88.2 \%(n=165)$ and 77.5\% $(n=145)$, respectively. The frequency of high-risk parameters in this cohort of patients that contributed to the FRANCE- 2 scores is shown in Table 1. The median score was 2 and the highest score was 9. The c-index of FRANCE-2 score for predicting 30-day mortality was $0.793(p=0.009)$ and for 1-year mortality was $0.679(p=0.016)$. The mean survival time for patients with high FRANCE-2 scores (18.6 months) was significantly less than for patients with low (53.8 months) and moderate ( 53.6 months) scores ( $p=0.0004)$. The logistic EuroSCORE was poorly associated with mortality with a $c$-index of $0.605(p=0.346)$ and $0.616(p=0.11)$ for 30-day and 1 -year mortality respectively.

\section{Conclusions}

The FRANCE-2 risk score is predictive of medium-term as well as short-term survival after TAVI in a single UK centre clinical practice. The logistic EuroSCORE is a poor predictor of short- and mediumterm survival after TAVI. The presence of a high FRANCE-2 score $(>5)$ is associated with poor survival after TAVI. The use of the FRANCE-2 scoring system may be a useful additional tool for the heart multidisciplinary team (MDT) in identifying patients who will benefit least from TAVI. 


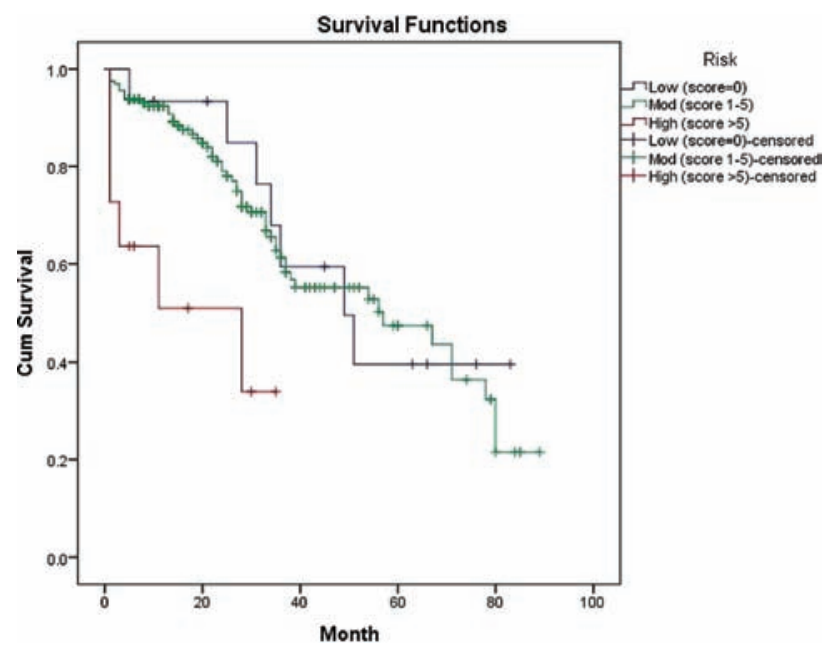

\section{Conflict of interest statement}

None declared.

\section{References}

1 Puri R, Iung B, Cohen D, Rodés-Cabau J. TAVI or No TAVI: identifying patients unlikely to benefit from transcatheter aortic valve implantation. European Heart Journal 2016;37:2217-25.

2 Iung B, Laouénan C, Himbert D et al. Predictive factors of early mortality after transcatheter aortic valve implantation: individual risk assessment using a simple score. Heart 2014;100:1016-23.

Fig 1. Survival functions 